\title{
Effects of Extracorporeal Shockwave Therapy on Improvements in Lymphedema, Quality of Life, and Fibrous Tissue in Breast Cancer-Related Lymphedema
}

Kyeong Woo Lee, MD, Sang Beom Kim, MD, Jong Hwa Lee, MD, Young Sam Kim, MD

Department of Physical Medicine and Rehabilitation, Dong-A University College of Medicine, Busan, Korea

Objective To evaluate the effects of extracorporeal shockwave therapy (ESWT) on improving lymphedema, quality of life, and fibrous tissue in patients with stage 2 lymphedema.

Methods Breast cancer-related lymphedema patients referred to the rehabilitation center were recruited. We enrolled stage 2 lymphedema patients who had firmness of the skin at their forearm, a circumference difference of more than $2 \mathrm{~cm}$ between each arm, or a volume difference between upper extremities greater than $200 \mathrm{~mL}$, confirmed by lymphoscintigraphy. The patients were randomly divided into the ESWT group and the control group. ESWT was performed for 3 weeks (two sessions per week); both groups received complex decongestive physical therapy. All patients were evaluated at baseline and at 3 weeks after treatment. The measurements performed included visual analog scale score, volume, circumference, QuickDASH (Quick Disabilities of the Arm, Shoulder and Hand) score, bioelectrical impedance, and skin thickness.

Results The patients in both groups ( $\mathrm{n}=15$ in each group) completed the 3 -week therapy experiment. No significant differences were observed in demographic characteristics between groups. After the 3-week treatment period, improvement was noted in the circumference difference below the elbow, volume, ratio of extracellular water to total body water, and skin thickness in the ESWT group. A significant difference was found in all the above-mentioned areas except in circumference below the elbow in the ESWT group.

Conclusion ESWT reduced edema and skin fibrosis without significant complications. Therefore, ESWT can be used together with complex decongestive physical therapy for treating lymphedema.

Keywords Extracorporeal shockwave therapy, Breast cancer lymphedema, Fibrosis, Electric impedance

Received December 11, 2019; Revised January 15, 2020; Accepted February 4, 2020; Published online September 28, 2020

Corresponding author: Young Sam Kim

Department of Physical Medicine and Rehabilitation, Dong-A University Hospital, Dong-A University College of Medicine, 26 Daesingongwon-ro, Seogu, Busan 49201, Korea. Tel: +82-51-240-5690, Fax: +82-51-254-8511, E-mail: ddoing3@naver.com

ORCID: Kyeong Woo Lee (http://orcid.org/0000-0001-8785-5535); Sang Beom Kim (http://orcid.org/0000-0002-5622-5933); Jong Hwa Lee (http:// orcid.org/0000-0003-2489-358X); Young Sam Kim (https://orcid.org/0000-0002-9156-0526).

(a) This is an open-access article distributed under the terms of the Creative Commons Attribution Non-Commercial License (http://creativecommons.org/ licenses/by-nc/4.0) which permits unrestricted noncommercial use, distribution, and reproduction in any medium, provided the original work is properly cited. Copyright ( 2020 by Korean Academy of Rehabilitation Medicine 


\section{INTRODUCTION}

Lymphedema is a chronic disease caused by damage to the lymphatic drainage system, resulting in protein accumulation, swelling, chronic inflammation, and fibrosis in tissues. Secondary lymphedema is caused by tumors of the lymph nodes, lymph node dissection, radiotherapy, trauma, or infection [1]. Upper limb lymphedema occurs in $24 \%-49 \%$ of mastectomy patients and $2.4 \%-49 \%$ of axillary lymphadenectomy patients [2]. According to the classification of the World Health Organization, the tissue initially becomes swollen in the form of non-pitting edema and then, in stage 2 , the affected limb becomes firm and enlarged. In stage 3, fibrosis worsens, resulting in irreversible changes in the tissues, which is due to secondary proliferation of neutrophils, macrophages, and fibroblasts and accumulation of collagen. A complex physical therapy regimen is the most widely used treatment for lymphedema.

The complex physical therapy consists of skin care, lymphatic drainage, compression tools, and exercise to promote lymph flow. Several studies demonstrated that complex physical therapy may reduce the volume of lymphedema by $21 \%-60 \%$ [3-7]. However, the therapy has the disadvantage of being affected by the therapist's technique and patients' compliance [8]. If there is progression beyond stage 2 , additional treatments may also be necessary for fibrotic tissue because the effect of therapy can be diminished due to fibrotic changes $[9,10]$.

Extracorporeal shockwave therapy (ESWT) is a recently developed non-invasive treatment approach that activates vascular endothelial growth factor (VEGF) and fibroblast, thereby promoting lymphatic neovascularization. Previous studies have reported that ESWT is effective in reducing lymphedema $[11,12]$. However, there is a lack of evidence on the effects of therapy according to the stage classification of disease and duration of treatment.
Therefore, the present study aimed to evaluate the effects of ESWT on the improvements of lymphedema, quality of life, and fibrous tissue in patients with stage 2 lymphedema.

\section{MATERIALS AND METHODS}

\section{Subjects}

The participants of this prospective study were female patients diagnosed with breast cancer by an oncology department who had visited an outpatient rehabilitation medical center for lymphedema treatment.

The present study enrolled stage 2 lymphedema patients with firmness at their forearm. For the objective evaluation of lymphedema, patients with circumference difference exceeding $2 \mathrm{~cm}$ between each arm and a volume difference over $200 \mathrm{~mL}$ (confirmed by lymphoscintigraphy) were included.

Patients without indication for ESWT due to bilateral, acute, and chronic inflammation as well as due to metastasis and poor skin condition were excluded. A total of 30 patients were randomly assigned to the ESWT and control groups.

\section{Methods}

For the ESWT group, extracorporeal shockwaves were generated using the electromagnetic type Dornier AR2 machine (Dornier MedTech, Wessling, Germany). The 1,000 times for the most fibrotic area and 1,500 times for the cubital lymph node and forearm area were performed twice a week for 3 weeks, a total of six times, at the strength of $0.056-0.068 \mathrm{~mJ} / \mathrm{mm}^{2}[13,14]$ (Fig. 1). For both groups, complex physical therapy such as manual lymphatic massage, specialized non-elastic bandage therapy, lymphatic drainage exercises, and skin care were commonly performed. All patients were treated by the same doctor and physiotherapist. To maintain patients'
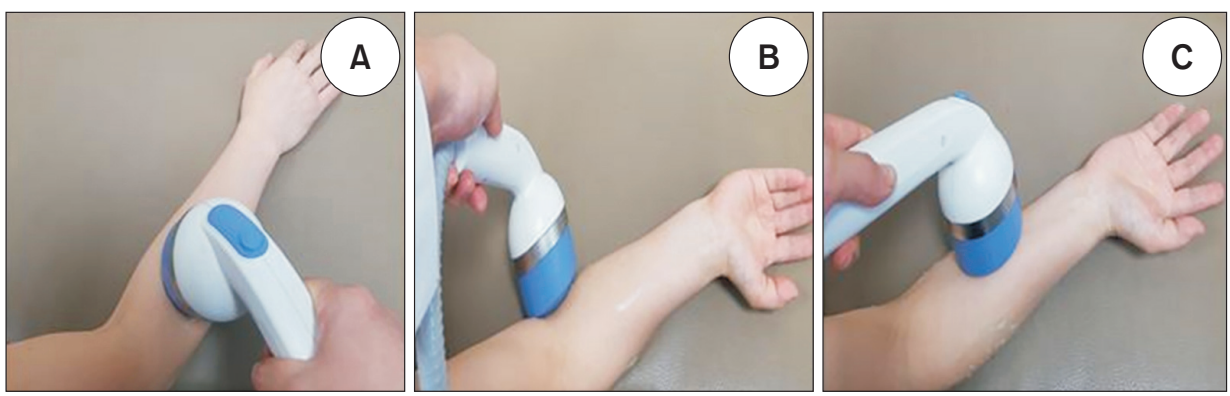

Fig. 1. (A) Extracorporeal shockwave therapy (ESWT) was applied to the most fibrotic lesion in the forearm. (B) ESWT was applied at the cubital lymph nodes. (C) ESWT was applied around the forearm. 
compliance, patient education was provided at every session.

\section{Assessment}

The patients were evaluated for each indicator twice: before treatment and at 3 weeks after treatment. Visual analog scale (VAS), with scores ranging from 0 to 10 , was used to measure the subjective pain degree. Lymphedema was evaluated by measuring the circumference of both arms and elbow, at $10 \mathrm{~cm}$ above and below the elbow and at the wrist and hand. For evaluating lymphedema, volume measurement was calculated as the amount of water remaining after the patient's arm was immersed to the axillary level in a water-filled cylinder.

Skin fibrosis, a complication of lymphedema, was evaluated by measuring the thickness using a skinfold caliper (Cambridge Scientific Industries Inc., Cambridge, MD, USA), and the mean value of three measurements of thickness of the most fibrotic lesion $10 \mathrm{~cm}$ below the elbow was used.

The Quick Disabilities of the Arm, Shoulder and Hand (QuickDASH) is a measurement of the functional status of the patient. This subjective assessment is conducted on items such as physical functional disability, pain, daily life difficulties, and muscle weakness, evaluating physical ability and symptoms of an arm with musculoskeletal disorder, ranging from 0 (no disability) to 100 (most severe disability). Subjective satisfaction of patients can be measured with this method.

The InBody S10 (Biospace Co. Ltd., Seoul, Korea) body composition analyzer was used to compare the ratio of extracellular water to total body water of the affected upper limb. A total of eight electrodes, two for each foot and hand, were used; the site for measurement was cleansed with alcohol before testing to reduce noise. The patients were examined in the supine position, and the test lasted for approximately 1 minute. As bioelectrical impedance analysis is sensitive to water content, 24 hours before the test, all activities that might affect water retention, such as extreme exercise or drinking, were restricted. The first and follow-up evaluations were performed at the same time of the day.

\section{Statistical analyses}

We used SPSS version 22.0 (IBM SPSS, Armonk, NY, USA) for conducting statistical analyses. To compare the

Table 1. Baseline characteristics of subjects at the initial evaluation

\begin{tabular}{|lccc}
\hline \multicolumn{1}{c}{ Characteristic } & Study $(\mathbf{n}=\mathbf{1 5})$ & Control $(\mathbf{n}=\mathbf{1 5})$ & p-value \\
\hline Age (yr) & $53.13 \pm 10.85$ & $52.24 \pm 8.60$ & 0.596 \\
\hline Sex, female & 15 & 15 & - \\
\hline Days from breast cancer-related surgery & $30.43 \pm 16.09$ & $28.30 \pm 11.17$ & - \\
\hline Duration of lymphedema (mo) & $12.83 \pm 8.21$ & $14.40 \pm 10.63$ & 0.142 \\
\hline Lymphedema stage 2 & 15 & 15 & - \\
Received chemotherapy & 15 & 15 & - \\
\hline Received radiotherapy & 12 & 13 & - \\
\hline VAS & $0.64 \pm 1.57$ & $0.52 \pm 1.35$ & 0.693 \\
\hline Circumference (cm) & & & \\
\hline Above elbow & $28.17 \pm 3.01$ & $26.81 \pm 4.28$ & 0.492 \\
\hline Elbow & $25.94 \pm 2.08$ & $24.44 \pm 2.37$ & 0.401 \\
\hline Below elbow & $26.28 \pm 3.02$ & $25.80 \pm 2.78$ & 0.556 \\
\hline Wrist & $16.21 \pm 0.90$ & $16.70 \pm 1.63$ & 0.761 \\
\hline Hand & $18.00 \pm 0.57$ & $17.90 \pm 1.28$ & 0.492 \\
Volume (mL) & $840.42 \pm 181.33$ & $822.00 \pm 144.68$ & 0.726 \\
\hline Rate of water content in upper extremity & $0.386 \pm 0.03$ & $0.380 \pm 0.02$ & 0.222 \\
\hline QuickDASH score & $4.75 \pm 5.72$ & $2.52 \pm 3.77$ & 0.426 \\
\hline Skin thickness (mm) & $31.14 \pm 2.91$ & $30.15 \pm 7.40$ & 0.510 \\
\hline
\end{tabular}

Values are presented as mean \pm standard deviation.

VAS, visual analog scale; QuickDASH, the Quick Disabilities of the Arm, Shoulder and Hand Questionnaire. 
results before and after treatment, statistical significance was confirmed with the Wilcoxon signed-rank test. For between-group analysis for significant differences in demographic characteristics and the parameters of test, the Mann-Whitney test was performed. Statistical significance was set at p-value below 0.05 .

\section{RESULTS}

A total of 30 patients with stage 2 lymphedema associated with breast cancer were included without dropouts. The patients were randomly divided into the ESWT group and the control group. In terms ofthe baseline characteristics of the two groups, all patients were female, had undergone modified radical mastectomy due to breast cancer, and had adjuvant chemotherapy and/or radiation.

The mean age of the ESWT group and the control group was $53.13 \pm 10.85$ and $52.24 \pm 8.60$ years, respectively. The mean duration of lymphedema was $12.83 \pm 8.21$ and $14.40 \pm 10.63$ months, respectively, and the duration of postoperative lymphedema was $30.43 \pm 16.09$ and $28.30 \pm 11.17$ months in the ESWT group and the control group, respectively. There were also no significant differences between the two groups in baseline characteristics including VAS score, circumference, volume, ratio of extracellular water to total body water, QuickDASH score, and skin thickness (Table 1).
Improvements were observed in both groups after 3 weeks of treatment (Table 2). In the ESWT group, the circumference (in $\mathrm{cm}$ ) below the elbow changed from $26.28 \pm 3.02$ to $25.50 \pm 3.12$ after the treatment ( $\mathrm{p}=0.026$ ), while the volume (in $\mathrm{mL}$ ) had changed from $840.42 \pm 181.33$ to $802.82 \pm 149.7$ ( $p=0.017)$. The ratio of extracellular water to total body water measured by bioelectrical impedance analysis also improved from $0.386 \pm 0.03$ to $0.379 \pm 0.01$ ( $\mathrm{p}=0.013$ ), and skin thickness (in $\mathrm{mm}$ ) measured by a skinfold caliper improved from $31.14 \pm 2.91$ to $29.85 \pm 3.09$ ( $\mathrm{p}=0.026)$.

The results of the comparison of changes in measurements between the two groups after 3 weeks of treatment are shown in Table 3. There was a significant difference in volume (\%) between the treatment group and the control group, $3.90 \pm 2.87$ and $1.85 \pm 1.89$, respectively $(\mathrm{p}=0.033)$. The changes in the ratio of extracellular water to total body water measured by bioelectrical impedance analysis for the ESWT and control groups were $0.007 \pm 0.02$ and $0.002 \pm 0.01$, respectively, indicating a significant difference $(p=0.031)$. There was also a significant difference in skin thickness $(\mathrm{mm})$ between the treatment group and the control group, $1.28 \pm 1.21$ and $0.61 \pm 1.45$, respectively $(\mathrm{p}=0.048)$.

Complications associated with ESWT, including soft tissue edema, hematoma, pain, and redness of the skin, were not observed in any of the patients during the study.

Table 2. Change of measurements between both groups after 3-week therapy

\begin{tabular}{|c|c|c|c|c|c|c|}
\hline & \multicolumn{3}{|c|}{ Study $(n=15)$} & \multicolumn{3}{|c|}{ Control $(n=15)$} \\
\hline & Pre & Post & p-value & Pre & Post & p-value \\
\hline VAS & $0.64 \pm 1.27$ & $0.43 \pm 0.78$ & 0.180 & $0.52 \pm 1.35$ & $0.40 \pm 0.84$ & 0.655 \\
\hline \multicolumn{7}{|l|}{ Circumference $(\mathrm{cm})$} \\
\hline Above elbow & $28.17 \pm 3.01$ & $27.14 \pm 4.46$ & 0.066 & $26.81 \pm 4.28$ & $25.55 \pm 2.65$ & 0.564 \\
\hline Elbow & $25.94 \pm 2.08$ & $25.63 \pm 3.14$ & 0.102 & $24.44 \pm 2.37$ & $24.04 \pm 2.07$ & 0.065 \\
\hline Below elbow & $26.28 \pm 3.02$ & $25.50 \pm 3.12$ & $0.026^{*}$ & $25.80 \pm 2.78$ & $25.40 \pm 2.19$ & 0.286 \\
\hline Wrist & $16.21 \pm 0.90$ & $16.00 \pm 0.76$ & 0.083 & $16.70 \pm 1.63$ & $16.55 \pm 1.53$ & 0.593 \\
\hline Hand & $18.00 \pm 0.57$ & $17.43 \pm 0.97$ & 0.102 & $17.90 \pm 1.28$ & $17.75 \pm 1.78$ & 0.450 \\
\hline Volume (mL) & $840.42 \pm 181.33$ & $802.80 \pm 149.7$ & $0.017^{*}$ & $822.00 \pm 144.68$ & $810.00 \pm 156.90$ & 0.469 \\
\hline Rate of water content in upper extremity & $0.386 \pm 0.03$ & $0.379 \pm 0.01$ & $0.013^{*}$ & $0.380 \pm 0.02$ & $0.378 \pm 0.01$ & 0.285 \\
\hline QuickDASH score & $4.25 \pm 5.72$ & $3.89 \pm 4.41$ & 0.317 & $3.15 \pm 4.28$ & $3.11 \pm 3.98$ & 0.987 \\
\hline Skin thickness (mm) & $31.14 \pm 2.91$ & $29.85 \pm 3.09$ & $0.026^{*}$ & $30.15 \pm 7.40$ & $29.54 \pm 6.98$ & 0.089 \\
\hline
\end{tabular}

Values are presented as mean \pm standard deviation.

VAS, visual analog scale; QuickDASH, the Quick Disabilities of the Arm, Shoulder and Hand Questionnaire.

${ }^{*} \mathrm{p}<0.05$ by Wilcoxon signed-rank test. 
Table 3. Comparison of changes between two groups

\begin{tabular}{lccc}
\hline & Study $(\mathbf{n}=\mathbf{1 5})$ & Control $(\mathbf{n}=\mathbf{1 5})$ & p-value \\
\hline$\Delta$ VAS & $0.21 \pm 0.78$ & $0.12 \pm 1.47$ & 0.294 \\
\hline Circumference $(\mathrm{cm})$ & & & \\
\hline$\Delta$ Above elbow & $1.02 \pm 1.07$ & $1.26 \pm 0.28$ & 0.255 \\
\hline$\Delta$ Elbow & $0.31 \pm 0.47$ & $0.40 \pm 0.55$ & 0.463 \\
\hline Below elbow & $0.78 \pm 0.63$ & $0.40 \pm 1.02$ & 0.273 \\
\hline Wrist & $0.21 \pm 0.26$ & $0.15 \pm 0.74$ & 0.328 \\
\hline Hand & $0.57 \pm 0.78$ & $0.15 \pm 0.58$ & 0.322 \\
\hline Volume (\%) & $3.90 \pm 2.87$ & $1.85 \pm 1.89$ & $0.033^{*}$ \\
$\Delta$ Rate of water content in upper extremity & $0.007 \pm 0.02$ & $0.002 \pm 0.01$ & $0.031^{*}$ \\
\hline$\Delta$ QuickDASH score & $0.35 \pm 2.26$ & $0.04 \pm 0.54$ & 0.065 \\
\hline Skin thickness (mm) & $1.28 \pm 1.21$ & $0.61 \pm 1.45$ & $0.048^{*}$ \\
\hline
\end{tabular}

Values are presented as mean \pm standard deviation.

VAS, visual analog scale; QuickDASH, the Quick Disabilities of the Arm, Shoulder and Hand Questionnaire.

${ }^{*} \mathrm{p}<0.05$ by Mann-Whitney U-test.

\section{DISSCUSSION}

The present study aimed to investigate the effects of ESWT on lymphedema and fibrotic lesions in breast cancer-associated lymphedema. According to the results, significant improvements in below elbow circumference, volume, ratio of extracellular water to total body water and skin thickness were observed in the ESWT group, and significant differences were found in volume, ratio of extracellular water to total body water, and skin thickness when comparing changes between the ESWT group and the control group.

Breast cancer is one of the most common tumors in women [15]. Secondary lymphedema is a chronic lifelong complication that occurs after breast cancer surgery and radiation therapy [16]. Lymphedema results from the accumulation of interstitial fluid due to damaged lymphatic drainage. Chronic lymphatic stasis accumulates fibroblasts, adipocytes, keratinocytes, and neutrophils, which, in turn, promotes the accumulation of collagen. Complex decongestive physical therapy, including manual lymphatic drainage, medical compression therapy, exercise, and skin care, is currently used as the first-line therapy for lymphedema, and several studies have demonstrated that it is effective in treating lymphedema associated with breast cancer $[7,17]$. The treatment duration of complex decongestive physical therapy may vary across patients, ranging from months to lifetime. The effect of this therapy is largely influenced by the degree of lymphedema, treatment methods, patient education, and compliance. Depending on the condition, continuous treatment for lifetime may be required, which may lead to a lower patient compliance, while increasing the cost burden $[9,18]$.

For these limitations, ESWT could be suggested as an additional therapy. In a previous study, ESWT was conducted in 7 patients with stage 3 lymphedema associated with breast cancer. Significant improvements in VAS score, volume, circumference, and skin thickness were observed after treatment [13]. In addition, in a study conducted by Cebicci et al. [14], the use of ESWT in breast cancer-related lymphedema patients showed significant improvements in volume, QuickDASH, and the brief version of the World Health Organization Quality of Life (WHOQOL-BREF). ESWT can stimulate the release of VEGF, which plays an essential role in promoting lymphangiogenesis, thereby relieving lymphedema. Previous human and animal studies showed that ESWT upregulates lymphangiogenesis and decreases inflammation $[11,12]$. Therefore, the effects on lymphedema found in the present study could be also seen as a result of improved lymphatic drainage by lymphangiogenesis.

Skin thickness was also evaluated in this study, which showed a reduction in the fibrotic changes in skin in patients with complaints of lymphedema associated with breast cancer. Untreated lymph accumulation causes lymphostatic fibrosis, which creates a snowball effect, causing more swelling. Therefore, as lymphedema stage worsens, fibrosis deteriorates. The results of a study 
in which ESWT were applied to patients with sclerosis demonstrated that ESWT may be an effective treatment for patients with skin fibrosis [19]. In the present study, the ESWT group also showed significant post-treatment improvements in skin thickness. Improvements in skin thickness found with skinfold caliper imply improvements in fibrosis such as improved skin elasticity and connective tissue strength [13].

While there are several methods for identifying lymphedema, including limb circumference measurement, water volume, and lymphoscintigraphy, there is no definite tool with both high sensitivity and specificity. Among other methods, bioelectrical impedance analysis is an easy-to-use, simple, inexpensive, and non-invasive tool to measure body composition [20,21]. Cornish et al. [22] demonstrated that lymphedema can be diagnosed according to bioelectrical impedance analysis in patients with $>3$ standard deviations compared to healthy controls. Furthermore, Ward and his colleagues $[23,24]$ identified lymphedema using bioelectrical impedance analysis and reported that these results are accurate and that therapeutic monitoring could be performed. Therefore, in the present study, bioelectrical impedance analysis was also used, and significant improvements were observed in changes when comparing the ESWT group and the control group.

The major goal of treating lymphedema is to reduce its effects. In the present study, ESWT was shown to be effective in the treatment of stage 2 lymphedema and skin fibrosis that occurred with the progression of this condition. The effects of ESWT on lymphedema were confirmed by decreased volume, rate of water content in the upper extremity, and skin thickness.

The present study has several limitations such as the small sample size. Therefore, further research including more patient groups will be needed. Furthermore, there could have been a selection bias, as only patients with stage 2 lymphedema were included in the sample. Therefore, it was difficult to derive meaningful results in VAS and QuickDASH scores. In addition, although the shortterm effects of ESWT could be evaluated in this study, further assessments to identify the long-term effects are needed. Finally, according to previous studies, a skinfold caliper was used to measure fibrosis, a complication of lymphedema [13]. However, this method has the limitation that the amount of fibrosis cannot be precisely de- termined. Several previous studies have used a variety of methods to measure fibrosis, but there is no current gold standard, warranting further research.

In summary, we provide evidence that ESWT can improve stage 2 breast cancer-related lymphedema. Significant improvement was observed in bioelectrical impedance analysis, volume, and skin thickness. Moreover, the results showed that that the therapy is effective on fibrotic lesions that cannot be effectively treated by complex decongestive physical therapy alone. Therefore, ESWT is a useful adjunct option for rehabilitation therapy that can complement conventional complex decongestive physical therapy.

\section{CONFLICT OF INTEREST}

No potential conflict of interest relevant to this article was reported.

\section{AUTHOR CONTRIBUTION}

Conceptualization: Lee KW. Methodology: Lee KW, Kim SB, Kim YS. Formal analysis: Lee JH. Project administration: Kim SB, Kim YS. Visualization: Lee KW, Lee JH, Kim YS. Writing - original draft: Lee KW, Kim YS. Writing review and editing: Kim YS. Approval of final manuscript: all authors

\section{REFERENCES}

1. Warren AG, Brorson H, Borud LJ, Slavin SA. Lymphedema: a comprehensive review. Ann Plast Surg 2007;59:464-72.

2. Hinrichs CS, Watroba NL, Rezaishiraz H, Giese W, Hurd T, Fassl KA, et al. Lymphedema secondary to postmastectomy radiation: incidence and risk factors. Ann Surg Oncol 2004;11:573-80.

3. Williams AF, Vadgama A, Franks PJ, Mortimer PS. A randomized controlled crossover study of manual lymphatic drainage therapy in women with breast cancer-related lymphoedema. Eur J Cancer Care (Engl) 2002;11:254-61.

4. Moattari M, Jaafari B, Talei A, Piroozi S, Tahmasebi S, Zakeri Z. The effect of combined decongestive therapy and pneumatic compression pump on lymphedema indicators in patients with breast cancer related 
lymphedema. Iran Red Crescent Med J 2012;14:210-7.

5. Hwang JM, Hwang JH, Kim TW, Lee SY, Chang HJ, Chu IH. Long-term effects of complex decongestive therapy in breast cancer patients with arm lymphedema after axillary dissection. Ann Rehabil Med 2013;37:690-7.

6. Finnane A, Janda M, Hayes SC. Review of the evidence of lymphedema treatment effect. Am J Phys Med Rehabil 2015;94:483-98.

7. Koul R, Dufan T, Russell C, Guenther W, Nugent Z, Sun X, et al. Efficacy of complete decongestive therapy and manual lymphatic drainage on treatment-related lymphedema in breast cancer. Int J Radiat Oncol Biol Phys 2007;67:841-6.

8. Tiwari P, Coriddi M, Salani R, Povoski SP. Breast and gynecologic cancer-related extremity lymphedema: a review of diagnostic modalities and management options. World J Surg Oncol 2013;11:237.

9. Yamamoto R, Yamamoto T. Effectiveness of the treatment-phase of two-phase complex decongestive physiotherapy for the treatment of extremity lymphedema. Int J Clin Oncol 2007;12:463-8.

10. Korpan MI, Crevenna R, Fialka-Moser V. Lymphedema: a therapeutic approach in the treatment and rehabilitation of cancer patients. Am J Phys Med Rehabil 2011;90(5 Suppl 1):S69-75.

11. Kubo M, Li TS, Kamota T, Ohshima M, Shirasawa B, Hamano K. Extracorporeal shock wave therapy ameliorates secondary lymphedema by promoting lymphangiogenesis. J Vasc Surg 2010;52:429-34.

12. Serizawa F, Ito K, Matsubara M, Sato A, Shimokawa $\mathrm{H}$, Satomi S. Extracorporeal shock wave therapy induces therapeutic lymphangiogenesis in a rat model of secondary lymphoedema. Eur J Vasc Endovasc Surg 2011;42:254-60.

13. Bae H, Kim HJ. Clinical outcomes of extracorporeal shock wave therapy in patients with secondary lymphedema: a pilot study. Ann Rehabil Med 2013;37:22934.

14. Cebicci MA, Sutbeyaz ST, Goksu SS, Hocaoglu S, Oguz A, Atilabey A. Extracorporeal shock wave therapy for breast cancer-related lymphedema: a pilot study.
Arch Phys Med Rehabil 2016;97:1520-5.

15. Mutlu H, Akca Z, Teke HU, Ugur H. Evaluation of peripheral blood smear for myelodysplasia in breast cancer patients who received adjuvant antracycline. Eurasian J Med 2011;43:173-6.

16. Horan D, McMullen M. Assessment and management of the woman with lymphedema after breast cancer. J Am Acad Nurse Pract 1998;10:155-9.

17. Vignes S, Porcher R, Arrault M, Dupuy A. Long-term management of breast cancer-related lymphedema after intensive decongestive physiotherapy. Breast Cancer Res Treat 2007;101:285-90.

18. Casley-Smith JR, Casley-Smith JR. Treatment of lymphedema by complex physical therapy, with and without oral and topical benzopyrones: what should therapists and patients expect. Lymphology 1996;29:76-82.

19. Tinazzi E, Amelio E, Marangoni E, Guerra C, Puccetti A, Codella OM, et al. Effects of shock wave therapy in the skin of patients with progressive systemic sclerosis: a pilot study. Rheumatol Int 2011;31:651-6.

20. Gupta D, Lammersfeld CA, Vashi PG, King J, Dahlk SL, Grutsch JF, et al. Bioelectrical impedance phase angle as a prognostic indicator in breast cancer. BMC Cancer 2008;8:249.

21. Tuorkey MJ. Bioelectrical impedance as a diagnostic factor in the clinical practice and prognostic factor for survival in cancer patients: prediction, accuracy and reliability. J Biosens Bioelectron 2012;3:121.

22. Cornish BH, Chapman M, Hirst C, Mirolo B, Bunce IH, Ward LC, et al. Early diagnosis of lymphedema using multiple frequency bioimpedance. Lymphology 2001;34:2-11.

23. Ward LC, Dylke E, Czerniec S, Isenring E, Kilbreath SL. Confirmation of the reference impedance ratios used for assessment of breast cancer-related lymphedema by bioelectrical impedance spectroscopy. Lymphat Res Biol 2011;9:47-51.

24. Ward LC. Bioelectrical impedance analysis: proven utility in lymphedema risk assessment and therapeutic monitoring. Lymphat Res Biol 2006;4:51-6. 